\title{
Correction to: Magnetic resonance features and cranial nerve involvement in pediatric head and neck rhabdomyosarcomas
}

\author{
Giacomo Talenti $^{1}$ - Stefania Picariello ${ }^{2,3} \cdot$ Caroline Robson $^{4} \cdot$ Livja Mertiri $^{5,6} \cdot$ Carmela Russo $^{7} \cdot$ Olga Slater $^{3}$. \\ Sotirios Bisdas ${ }^{8} \cdot$ Massimo Eraldo Abate $^{9} \cdot$ Silverio Perrotta $^{2} \cdot$ Richard Hewitt $^{10} \cdot$ Kshitij Mankad $^{5} \cdot$ Felice D'Arco $^{5}$
}

Published online: 6 September 2021

(c) Springer-Verlag GmbH Germany, part of Springer Nature 2021

\section{Correction to: Neuroradiology (2021) \\ https://doi.org/10.1007/s00234-021-02765-0}

Originally, the article has been published online with inverted author names. This has been corrected above.

The original article has been corrected.

Publisher's note Springer Nature remains neutral with regard to jurisdictional claims in published maps and institutional affiliations.

The original article can be found online at https://doi.org/10.1007/ s00234-021-02765-0.

Giacomo Talenti

giacomo.talenti@aovr.veneto.it

1 Neuroradiology Unit, Department of Diagnostics and Pathology, Verona University Hospital, Azienda Ospedaliera Universitaria Integrata (AOUI) Di Verona, Piazzale Aristide Stefani 1, Verona, Italy

2 Department of Woman, Child and General and Specialized Surgery, University of Campania Luigi Vanvitelli, Naples, Italy

3 Haematology and Oncology Department, Great Ormond Street Hospital for Children, London, UK

4 Division of Neuroradiology, Department of Radiology, Children's Hospital, Harvard Medical School, Boston, MA, USA
5 Neuroradiology Department, Great Ormond Street Hospital for Children, London, UK

6 Faculty of Medicine and Dentistry, Sapienza University of Rome, Rome, Italy

7 Neuroradiology Department, Santobono Children's Hospital, Naples, Italy

8 Lysholm Department of Neuroradiology, The National Hospital for Neurology and Neurosurgery, Queen Square 8-11, London WC1N 3BG, UK

9 Pediatric Oncology, AORN Santobono-Pausilipon, Napoli, Italy

10 Ear, Nose and Throat Surgery Department, Great Ormond Street Hospital for Children NHS Trust, London, UK 